\title{
Estrutura de comunidade da quiropterofauna (Mammalia, Chiroptera) do Parque Estadual de Campinhos, Paraná, Brasil
}

\author{
Ives S. Arnone ${ }^{1,3} \&$ Fernando C. Passos ${ }^{2,3}$ \\ ${ }^{1}$ Departamento de Zoologia, Instituto de Biociências, Universidade de São Paulo. Caixa Postal 11461, 05422970 São \\ Paulo, São Paulo, Brasil. E-mail: ives@usp.br \\ ${ }^{2}$ Laboratório de Biodiversidade, Conservação e Ecologia de Animais Silvestres, Departamento de Zoologia, Universidade \\ Federal do Paraná. Caixa Postal 19020,81531-980, Curitiba, Paraná, Brasil. E-mail: fpassos@ufpr.br \\ ${ }^{3}$ Bolsista do CNPq.
}

\begin{abstract}
Community Structure of Chiropterofauna (Mammalia, Chiroptera) of Campinhos State Park, Paraná, Brazil. This study presents information about a survey of bats made in Campinhos State Park (PEC) ( $25^{\circ} \mathrm{O} 2^{\prime} \mathrm{S}, 49^{\circ} \mathrm{O} 5^{\prime} \mathrm{W}, 890 \mathrm{~m}$ altitude), Paraná, Brazil. This Conservation Unit encompasses 336.97 ha. The main tourist attractions are the caves known as Jesuitas/Fada. PEC is located at $63 \mathrm{~km}$ north of Curitiba. Different stages of Araucaria Pine Forest characterize the park vegetation. Twelve field trips had been carried monthly from September 2003 to August 2004 using 10 mist nets. In this period, 423 bats were captured, 274 Desmodus rotundus (E. Geoffroy, 1810$)(64.8 \%)$ and 50 Myotis nigricans (Schinz, 1821) (11.8\%), both representing $76.6 \%$ of the survey. Other 12 species were identified, like Anoura caudifer (E. Geoffroy, 1818); Anoura geoffroyi Gray, 1838; Carollia perspicillata (Linnaeus, 1758); Chrotopterus auritus (Peters, 1856); Diphylla ecaudata Spix, 1823; Eptesicus furinalis (d'Orbigny, 1847); Eptesicus taddeii (Miranda et al., 2006); Glossophaga soricina (Pallas, 1766); Lasiurus blossevillii (Lesson \& Garnot, 1826); Mimon bennettii (Gray, 1838); Pygoderma bilabiatum (Wagner, 1843) and Sturnira lilium (E. Geoffroy, 1810). Some of the species considered threatened in Paraná State, such as C. auritus, D. ecaudata and M. bennettii, were all captured in grottos.
\end{abstract}

KEY WORDS. Bats; caves; shelter.

RESUMO. Este estudo apresenta informações sobre a quiropterofauna do Parque Estadual de Campinhos (PEC) ( $25^{\circ} \mathrm{O} 2^{\prime} \mathrm{S}, 49^{\circ} \mathrm{O} 5^{\prime} \mathrm{W}, 890 \mathrm{~m}$ altitude), Paraná. Esta Unidade de Conservação possui 336,97 ha. Seu maior atrativo turístico são as cavernas Jesuítas/Fada. O PEC situa-se a $63 \mathrm{~km}$ ao norte de Curitiba. A vegetação é marcada por diferentes estágios sucessionais da Floresta Ombrófila Mista. Doze saídas mensais foram realizadas entre setembro de 2003 e agosto de 2004, utilizando-se 10 redes-de-neblina. Nesse período, 423 morcegos foram capturados, dos quais 274 foram Desmodus rotundus (E. Geoffroy, 1810) (64,8\%) e 50 foram Myotis nigricans (Schinz, 1821) $(11,8 \%)$ ambos representando $76,6 \%$ da amostragem. Além dessas espécies, outras 12 foram identificadas como Anoura caudifer (E. Geoffroy, 1818); Anoura geoffroyi Gray, 1838; Carollia perspicillata (Linnaeus, 1758); Chrotopterus auritus (Peters, 1856); Diphylla ecaudata Spix, 1823; Eptesicus furinalis (d'Orbigny, 1847); Eptesicus taddeii (Miranda et al., 2006); Glossophaga soricina (Pallas, 1766); Lasiurus blossevillii (Lesson \& Garnot, 1826); Mimon bennettii (Gray, 1838); Pygoderma bilabiatum (Wagner, 1843) e Sturnira lilium (E. Geoffroy, 1810). Algumas das espécies consideradas vulneráveis no Estado do Paraná como C. auritus, D. ecaudata e M. bennettii foram capturadas nas grutas.

PALAVRAS-CHAVE. Abrigos diurnos; cavernas; morcegos.

A ordem Chiroptera é umas das ordens dominantes de mamíferos na Região Neotropical. Tradicionalmente a ordem é dividida em duas subordens, Megachiroptera e Microchiroptera. A última é amplamente distribuída pelo mundo, sendo representada por 17 famílias, 157 gêneros e 928 espécies (Simmons 2005, Peracchi et al. 2006). São 164 espécies com ocorrência para o Brasil (Reis et al. 2006), representando cerca de um terço da mastofauna do País (Fonseca et al. 1999). Esta diversidade deve-se em grande parte à variedade de hábitos alimentares como insetivoria, frugivoria, nectarivoria, carnivoria, piscivoria, sanguivoria e onivoria encontrados em membros da família Phyllostomidae, os quais representam aproximadamente 55\% da fauna Neotropical (Humphrey \& Bonaccorso 1979).

No Brasil, 42 espécies de morcegos já foram registradas vivendo em cavernas, o que representa pouco mais de $25 \%$ do número total de espécies (dados compilados de PINTO-DA-RochA 
1995, Trajano \& Gimenez 1998, Gregorin \& Mendes 1999, Silva et al. 2001, Zeppelini et al. 2003). No País, alguns trabalhos sobre riqueza de quirópteros em cavernas já foram desenvolvidos, como os de Silva et al. (2001) no Estado do Ceará, Gregorin \& Mendes (1999) no Estado da Bahia, Bredt et al. (1999) no Distrito Federal, Trajano \& Gimenez (1998) no Estado de Minas Gerais, Trajano $(1984,1995,1996)$, Campanhã \& Fowler (1995) e Zeppelini et al. (2003) no Estado de São Paulo, e finalmente Oliveira \& SiPInski (2001) no Estado do Paraná. As cavernas com maiores dimensões fornecem grande proteção para morcegos, não só em função da estabilidade climática, mas também pela raridade de outros mamíferos, aves, répteis ou artrópodes especializados que poderiam atuar como predadores ou competidores nesses locais (Trajano 1984). Devido a essas características do ambiente cavernícola, muitas espécies tendem a formar grandes colônias, podendo haver coabitação com outras espécies (Kunz 1982, Trajano 1984).

No Estado do Paraná foram registradas 53 espécies de morcegos, das quais 36 foram observadas na formação Floresta Ombrófila Mista (Miretzki 2003). Recentemente dois novos registros de morcegos foram obtidos para o Estado (Miranda et al. 2006a, 2007) e uma nova espécie do gênero Eptesicus foi descrita (Miranda et al. 2006b). Tais registros elevam o número total para 56 espécies. Há registros de morcegos cavernícolas em 26 cavernas no Estado do Paraná, sendo estas ocupadas por apenas sete espécies (PINTO-DA-Rocha 1995, OliveIRA \& SiPINSKI 2001), o que reflete o pouco conhecimento e estudo da quiropterofauna cavernícola na região. Os estudos com quirópteros ainda são incipientes, embora crescentes, e mesmo aspectos mais básicos do estudo da biodiversidade, como as listas de espécies, inexistem ou estão desatualizadas para quase todo o território brasileiro (MiretzKi 2003).

O patrimônio espeleológico paranaense já se encontra em grande parte destruído, sendo as principais causas dessa degradação a exploração mineral sem controle, o turismo desordenado, a expansão urbana, e a poluição hídrica (SESSEGOLO et al. 1996). Estima-se que no mínimo 50\% do patrimônio espeleológico da Região Metropolitana de Curitiba encontrase hoje destruído, sem nem mesmo ter sido estudado (Grupo de Estudos Espeleológicos do Paraná/GEEP-Açungui, dados não publicados). O PEC localiza-se na Região Metropolitana de Curitiba e foi criado em 1960, com o objetivo principal de proteger as cavernas do Conjunto Jesuítas/Fada (PR-0009). O presente trabalho teve como objetivo estudar a comunidade de morcegos que ocorre no PEC, a fim de contribuir com o plano de manejo dessa Unidade de Conservação, e com a biologia e conservação de morcegos cavernícolas do Estado do Paraná.

\section{MATERIAL E MÉTODOS}

\section{Área de Estudo}

O Parque Estadual de Campinhos (PEC) $\left(25^{\circ} 02^{\prime} \mathrm{S}, 49^{\circ} 05^{\prime} \mathrm{W}\right)$ localiza-se na porção norte da zona fisiográfica do Primeiro Planalto Paranaense, inserindo-se nos municípios de Tunas do
Paraná e Cerro Azul, distando cerca de 63 km ao norte de Curitiba e 8 km de Tunas do Paraná (IAP 2000). Esta Unidade de Conservação apresenta hoje uma área de 336,97 ha, e tem como seu maior atrativo as grutas do Conjunto Jesuítas/Fada.

O PEC encontra-se em uma altitude de 890 m sendo situado na Bacia Hidrográfica do Rio Ribeira. Segundo a classificação climática de Köppen, o clima da região pode ser caracterizado como pertencente ao tipo Cfb: sem estação seca definida e com ocorrências de geadas severas e freqüentes, com precipitação média anual variando entre 1400 a $1500 \mathrm{~mm}$, sendo de $1675 \mathrm{~mm}$ no ano do presente estudo (IAPAR 2006). A vegetação é marcada pela presença da Floresta Ombrófila Mista Montana de acordo com Veloso et al. (1991), apresentando hoje diferentes estágios sucessionais. $\mathrm{O}$ acesso principal ao parque se faz pela rodovia federal BR-476, mais conhecida como Estrada da Ribeira (Fig. 1).

O PEC foi criado em 1960 com objetivo principal de proteger as cavernas presentes na região. Nas décadas de 40 e 50 houve uma intensa exploração de madeiras de lei, de lenha, ou pela conversão das terras para fins agropecuários na área, restando poucos remanescentes florestais primários. Na década de 60 , o aspecto fitofisionômico da região era resultante de uma intensa e descontrolada intervenção humana ocasionada pelas décadas anteriores. Atualmente no PEC, bem como em sua região de entorno, verificam-se alterações conseqüentes do intenso processo de transformação da cobertura vegetal. A vegetação nativa foi substituída pela atividade agropastoril, mineração de calcário, e além destas, a exploração seletiva de madeira, onde mais recentemente sobressaem-se os reflorestamentos de Pinus elliottii e Pinus taeda. (Geep-Açungui \& Iap 2003).

\section{Sítios de Estudo}

As cavernas do PEC foram exploradas e topografadas pelos membros do Grupo de Estudos Espeleológicos do Paraná, GEEP-AçUNGUI, e os mapas encontram-se no cadastro dessa entidade.

PR-0009. O Conjunto Jesuítas/Fada $\left(25^{\circ} 02^{\prime} 20,4^{\prime \prime}\right.$, $49^{\circ} 05^{\prime} 30,4^{\prime \prime} \mathrm{W}$ ) é composto pelas Grutas dos Jesuítas e da Fada que somadas possuem $1.527 \mathrm{~m}$ de extensão (desenvolvimento) e um desnível de $38 \mathrm{~m}$. O conjunto apresenta sete entradas, das quais quatro foram amostradas (Sumidouro, Ressurgência, Abismo Professor Schiebler e Fada). A gruta dos Jesuítas é a quinta maior cavidade do Estado com 1419 m de desenvolvimento, apresentando rio corrente de pequeno porte.

A Gruta da Fada (108 m de desenvolvimento) e o Abismo Professor Schiebler fazem parte da porção superior desse sistema cárstico, sendo que ambas encontram-se visualmente em equivalência de cotas de nível. Porém, tanto a Gruta da Fada como o Abismo, encontram-se interrompidos por um grande dolina, denominada Dolina da Fada, ambas sem rios. O Abismo Professor Schiebler possui uma ligação com as galerias da Gruta dos Jesuítas. Esta interligação se faz através de uma fenda estreita, enquanto que a Gruta da Fada encontra-se isolada do sistema da Gruta dos Jesuítas devido ao colapso da dolina (GEeP-AÇUNGUI \& IAP 2003).

Revista Brasileira de Zoologia 24 (3): 573-581, setembro 2007 


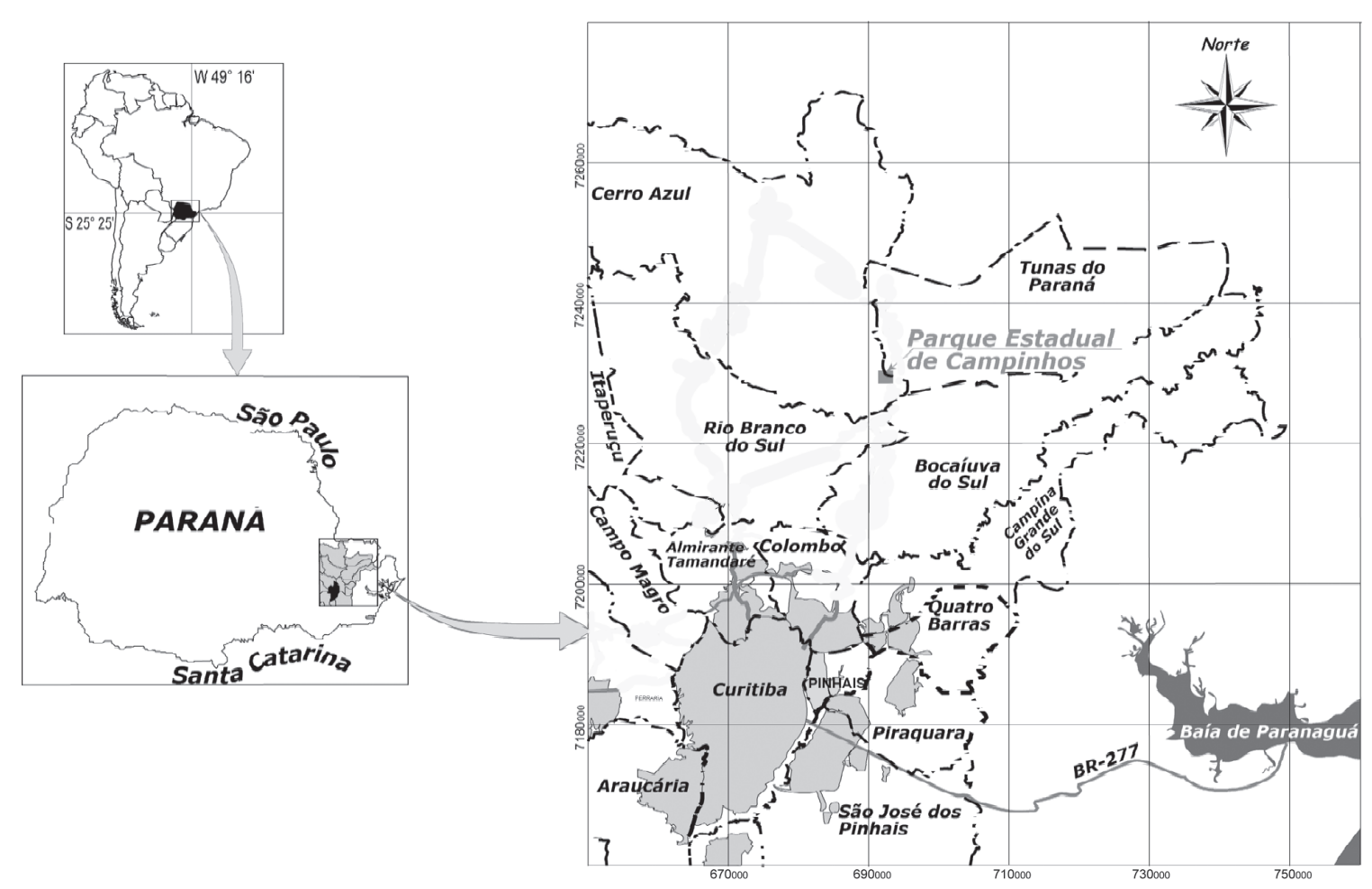

Figura 1. Mapa de localização do Parque Estadual de Campinhos. As linhas tracejadas indicam os limites municipais. Fonte: GeEp-AcunGuI $\&$ IAP (2003).

Mata. Na mata foram amostrados quatro pontos, três dos quais se situaram ao longo da trilha principal que dá acesso as duas entradas da caverna (Ressurgência e Sumidouro), enquanto que o último ponto se situou numa trilha secundária, também utilizada por turistas nas caminhadas. A distância entre essas redes foi aproximadamente de $200 \mathrm{~m}$.

\section{Método de amostragem}

O estudo foi realizado mensalmente, de setembro de 2003 a agosto de 2004, abrangendo as cavernas do Conjunto Jesuítas/Fada e as trilhas que representavam possíveis corredores de vôo dos animais, possibilitando a construção da curva do coletor e a análise da abundância de cada espécie por mês. Cada fase mensal de campo consistiu em duas noites de amostragem em lua minguante ou nova, cobrindo todo o período noturno, num total de 24 noites para o período de estudo. Ao longo do ano, as variações no comprimento da noite foram anotadas obtendo-se a duração aproximada de 9:40 h no mês de noite mais curta (janeiro) e 13:00 h na noite mais longa (julho). Isto representou, aproximadamente, as horas de amostragem de morcegos nas noites ao longo do estudo.

Foram utilizadas 10 redes de neblina (mist net) com dimensões de 6 a $10 \mathrm{~m}$ de comprimento por 2,5 m de altura. No caso das entradas de grande porte (Sumidouro e Abismo) foram dispostas duas redes, uma baixa e uma alta colocadas en- tre 0,5 e 4,0 m de altura do solo, respectivamente, num total de $40 \mathrm{~m}^{2}$ de rede por noite. Nas pequenas entradas (Fada e Ressurgência) foi utilizada apenas uma rede por local num total de $25 \mathrm{~m}^{2}$ de rede por noite. Nas entradas de grande porte o esforço mensal médio foi de 913 h.m², enquanto nas entradas de pequeno porte foi de 570 h.m². A composição da fauna de cada uma das aberturas foi agrupada e analisada através do índice de Jaccard. O método de agrupamento utilizado foi o UPGMA através do programa NTSYS. Nas trilhas, quatro redes foram armadas perfazendo um total de $100 \mathrm{~m}^{2}$ de redes por noite, numa média mensal de 2283 h.m². O total de esforço amostral no estudo foi de 63020 h.m ${ }^{2}$. Os dados de esforço foram calculados de acordo com Straube \& Bianconi (2002).

Os morcegos capturados foram acondicionados individualmente em sacos de algodão para posterior identificação taxonômica (em campo, quando possível). Para a identificação das espécies foram utilizadas chaves de Chiroptera de LAVAL (1973), Vizotto \& Taddei (1973), Barquez et al. (1993), Reis et al. (1993) e Lim \& Engstrom (2001). Em campo também foram realizadas a determinação de sexo, estágio de desenvolvimento (jovem ou adulto), condição reprodutiva no caso das fêmeas e finalmente anilhamento. Esses animais foram libertados no seu local de captura após o fechamento das redes. Foi montada uma coleção de material testemunho, com exemplares de cada espécie quando possível. No caso de espécies criptícas (como 
Myotis) um maior número de indivíduos foi coletado para uma identificação mais precisa. Tais exemplares foram depositados na Coleção Científica do Departamento de Zoologia da Universidade Federal do Paraná (DZUP), Curitiba, na Seção de Mastozoologia (Tab. I).

\section{RESULTADOS E DISCUSSÃO}

Foram capturados 423 morcegos (incluindo 125 recapturas) de 14 espécies, representadas por duas famílias, Phyllostomidae $(\mathrm{n}=11)$ e Vespertillionidae $(\mathrm{n}=3)$. Dessas capturas, 274 foram Desmodus rotundus $(64,8 \%)$ e 50 foram Myotis nigricans $(11,8 \%)$, ambos representando, portanto $76,6 \%$ da amostragem (Tab. I).

Os resultados da espécie mais abundante durante as amostragens no PEC (D. rotundus $=64,8 \%$ ), se assemelham aos resultados obtidos por Trajano (1984) nas cavernas do Vale do Ribeira, PETAR-SP (44,5\%), e por BREDT et al. (1999) nas cavernas da região do Distrito Federal (54,9\%). Apesar dessas semelhanças a dominância desta espécie no PEC foi mais evidente. OLIVEIRA \& SIPINSKI (2001), trabalhando nas cavernas da Região Metropolitana de Curitiba, também encontraram $D$. rotundus como a mais abundante, porém os dados não foram quantificados. Esses resultados comprovam a boa adaptação desses morcegos a se abrigarem em cavernas, que de acordo com CAMPANHA \& Fowler (1995) e Gomes \& Uieda (2004) costumam ser encontrados em grandes colônias. A grande abundância de $D$. rotundus no PEC pode ser reflexo de uma grande população da espécie na região, devido à alta disponibilidade de alimento, e da reprodução contínua.
A segunda espécie mais capturada foi $M$. nigricans com $11,8 \%$ de freqüência. Esse resultado é bem superior ao encontrado por TRAjANo (1984) e BREDT et al. (1999) que constataram freqüências de $4 \%$ e $0,6 \%$ respectivamente. O sucesso de captura dessa espécie no presente estudo foi provavelmente devido ao uso de redes num estrato mais elevado (Sumidouro e Abismo) e/ ou pelo fato das pequenas entradas (Fada e Ressurgência) terem sido quase totalmente bloqueadas pelas redes.

Anteriormente a este estudo, MiRETZKi (2003) reconheceu seis espécies para a área do PEC, sendo elas Molossus molossus (Pallas, 1766), Myotis levis (I. Geoffroy, 1824), Anoura caudifer, Chrotopterus auritus, Desmodus rotundus e Mimon bennettii. Com o presente trabalho, foram acrescidas 10 espécies para o PEC, elevando para 16 o número de quirópteros conhecidos na região. É de se destacar a ausência de capturas de Artibeus lituratus (Olfers, 1818), uma das espécies neotropicais mais bem sucedidas, citada por Trajano (1984) como uma das espécies de quirópteros mais comuns nas cavernas do Alto Ribeira. Uma das possíveis razões para a não ocorrência de $A$. lituratus e congêneres, seria a ausência nos locais amostrados de plantas das famílias Cecropiaceae e Moraceae, cujos frutos consistem em um recurso alimentar muito explorado por essas espécies (PAssos et al. 2003, Passos \& Graciolli 2004).

Nesse estudo, das 14 espécies encontradas no PEC, dez delas foram capturadas usando as cavernas como abrigo. As demais, como a espécie frugívora Pygoderma bilabiatum e os quirópteros insetívoros Lasiurus blossevillii, Eptesicus furinalis e

Tabela I. Morcegos capturados no PEC com seus respectivos hábitos alimentares e local de tombo.

\begin{tabular}{|c|c|c|c|c|}
\hline Família & Espécie & $\begin{array}{c}\text { Hábitos } \\
\text { alimentares }\end{array}$ & Local de tombo & Número \\
\hline \multirow[t]{11}{*}{ Phyllostomidae } & Anoura caudifer (E. Geoffroy, 1818) & Nectarívoro & $\mathrm{CCMZ-UFPR}^{1} / \mathrm{MHNCl}^{2}$ & DZUP 053, 115 \\
\hline & Anoura geoffroyi Gray, 1838 & Nectarívoro & CCMZ-UFPR & DZUP 052, 055 \\
\hline & Carollia perspicillata (Linnaeus, 1758) & Frugívoro & CCMZ-UFPR & DZUP 080 \\
\hline & Chrotopterus auritus (Peters, 1856) & Carnívoro & CCMZ-UFPR & DZUP 043 \\
\hline & Desmodus rotundus (E. Geoffroy, 1810) & Hematófago & CCMZ-UFPR/MHNCl${ }^{2}$ & DZUP 004, 005, 006 \\
\hline & Diphylla ecaudata Spix, 1823 & Hematófago & CCMZ-UFPR & DZUP 050,051 \\
\hline & Glossophaga soricina (Pallas, 1766) & Nectarívoro & CCMZ-UFPR & DZUP 007, 078 \\
\hline & Pygoderma bilabiatum (Wagner, 1843) & Frugívoro & CCMZ-UFPR & DZUP 054 \\
\hline & Mimon bennettii (Gray, 1838) & Insetívoro & CCMZ-UFPR/MHNCl${ }^{2}$ & DZUP 044, 045, 081 \\
\hline & Pygoderma bilabiatum (Wagner, 1843) & Frugívoro & CCMZ-UFPR & DZUP 054 \\
\hline & Sturnira lilium (E. Geoffroy, 1810) & Frugívoro & CCMZ-UFPR & DZUP 008, 079, 153, 154 \\
\hline \multirow[t]{4}{*}{ Vespertilionidae } & Eptesicus taddeii (Miranda et al., 2006) & Insetívoro & CCMZ-UFPR & DZUP 094, 098 \\
\hline & Lasiurus blossevillii (Lesson \& Garnot, 1826) & Insetívoro & CCMZ-UFPR & DZUP 113 \\
\hline & Myotis levis (I. Geoffroy, 1824) * & Insetívoro & $\mathrm{MHNCl} 2$ & \\
\hline & Myotis nigricans (Schinz, 1821) & Insetívoro & CCMZ-UFPR & $\begin{array}{l}\text { DZUP 001, 002, 056-059, } 061- \\
067,085-090,092,093,095- \\
097,099-112\end{array}$ \\
\hline Molossidae & Molossus molossus (Pallas, 1766) * & Insetívoro & $\mathrm{MHNCl}^{2}$ & \\
\hline
\end{tabular}

*) Animais não coletados nesse estudo; 1) Coleção Científica de Mastozoologia do Departamento de Zoologia da Universidade Federal do Paraná, DZUP; 2) Museu de História Natural Capão da Imbuia. Animais sem número tombo definitivo.

Revista Brasileira de Zoologia 24 (3): 573-581, setembro 2007 
E. taddei, foram capturadas exclusivamente em redes colocadas nas trilhas e possíveis corredores de vôo (Tab. II).

Segundo PINTO-DA-Rocha (1995), o PEC possuía o registro para quatro espécies de morcegos utilizando as cavernas do Conjunto Jesuítas/Fada: Anoura caudifer, Chrotopterus auritus, Desmodus rotundus e Mimon bennettii. As demais espécies, Diphylla ecaudata, Glossophaga soricina e Carollia perspicillata eram encontradas apenas em outras cavidades do Estado. Com o presente estudo, o PEC passa a ser a caverna com a maior riqueza de quirópteros para o Estado do Paraná (10 espécies).

Nas quatro entradas do Conjunto Jesuítas/Fada foram observadas diferenças na riqueza de espécies e nas suas curvas do coletor (Fig. 2). Tal fato seria uma evidência da utilização diferencial das quatro entradas pelas espécies amostradas, apesar de as entradas serem parte de uma mesma caverna, mas que funcionariam como abrigos distintos (Fig. 3). Nessa análise de agrupamento pode-se verificar que a composição de espécies de morcegos entre as entradas com e sem visitação pública se agruparam. Assim, Fada e Abismo, entradas sem a visitação pública apresentaram uma fauna semelhante, enquanto aquelas abertas à visitação pública (Ressurgência e Sumidouro), apresentaram uma composição de espécies de morcegos semelhantes entre si (Fig. 3). Isto pode refletir a maior proximidade entre a entrada e o local de ocupação no interior da caverna (Tab. III). Após cinco a oito meses de estudo, observamos que o número se estabilizou entre sete e oito espécies de morcegos para as quatro entradas amostradas (Fig. 2). Para a mata, após sete meses foi observado uma estabilização em 13 espécies, não sendo encontrada apenas Glossophaga soricina (Fig. 4).

Segundo Trajano (1995) e BREDT et al. (1999) a comunidade de morcegos cavernícolas é particularmente rica em espécies de subfamília Phyllostominae, porém isso não foi evidenciado no PEC, onde apenas duas espécies, Mimon bennettii e Chrotopterus auritus, foram encontradas. Willig \& SELCER (1989) e FABIÁn et al. (1999), assumem que a latitude e a riqueza do bioma são fatores determinantes na distribuição geográfica das espécies da família Phyllostomidae, e conseqüentemente de suas subfamílias. No Estado do Paraná, a Subfamília Phyllostominae é representada por seis espécies, Tonatia bidens (Spix 1823); Phyllostomus hastatus (Pallas, 1767); Macrophyllum macrophyllum (Schinz 1821); Micronycteris megalotis (Gray, 1838); Chrotopterus auritus e Mimon bennettii, sendo as quatro últimas encontradas na Floresta Ombrófila Mista (Miretzki 2003). Portanto, as duas espécies registradas equivalem a 50\% das encontradas em Floresta Ombrófila Mista para o Estado do Paraná, o que pode ser considerado uma riqueza razoável em uma única localidade.

Algumas espécies encontradas no PEC (C. auritus, D. ecaudata e $M$. bennettii) são consideradas vulneráveis, estando sob alto risco de extinção na natureza no Estado do Paraná (Margarido \& Braga 2004). Todas essas espécies foram encontradas nas cavernas do PEC, o que reforça a importância desses ambientes para a sua conservação. Isso corrobora com a afirmação de TrajANo (1995) que salienta a importância das cavernas na conservação de Phyllostominae.

Tabela III. Freqüência das espécies capturadas em cada uma das entradas estudadas.

\begin{tabular}{lcccc}
\hline \multicolumn{1}{c}{ Espécies } & Ressurgência & Sumidouro & Abismo & Fada \\
\hline Anoura caudifer & 1 & 2 & 13 & 1 \\
Anoura geoffroyi & & 2 & 2 & \\
Carollia perspicillata & 1 & 4 & 1 & \\
Chrotopterus auritus & 4 & 79 & 11 & 48 \\
Desmodus rotundus & 91 & & 1 & 1 \\
Diphylla ecaudata & 3 & 1 & 1 & 2 \\
Glossophaga soricina & & 11 & 24 & 2 \\
Mimom bennettii & 1 & 1 & & \\
Myotis nigricans & 5 & & & \\
Sturnira lilium & 1 & &
\end{tabular}

Tabela II. Morcegos capturados no PEC utilizando ou não cavernas na área como abrigo diurno, com suas respectivas freqüências de captura.

\begin{tabular}{|c|c|c|c|c|c|c|c|}
\hline Utilizaram as grutas como abrigo & Capturas* & Macho & Fêmea & Não utilizaram as grutas & Capturas & Macho & Fêmea \\
\hline Anoura caudifer & 23 & 15 & 8 & Eptesicus furinalis & 1 & 1 & 0 \\
\hline Anoura geoffroyi & 5 & 0 & 5 & Eptesicus taddeii & 2 & 0 & 2 \\
\hline Carollia perspicillata & 14 & 9 & 5 & Lasiurus blossevillii & 1 & 1 & 0 \\
\hline Chrotopterus auritus & 13 & 8 & 5 & Pygoderma bilabiatum & 9 & 2 & 7 \\
\hline Desmodus rotundus & $274^{* *}$ & 160 & 112 & & & & \\
\hline Diphylla ecaudata & 6 & 6 & 0 & & & & \\
\hline Glossophaga soricina & 3 & 2 & 1 & & & & \\
\hline Mimon bennettii & 10 & 3 & 7 & & & & \\
\hline Myotis nigricans & $50^{* * *}$ & 16 & 25 & & & & \\
\hline Sturnira Iilium & 12 & 10 & 2 & & & & \\
\hline Total & 410 & 229 & 170 & & 13 & 4 & 9 \\
\hline
\end{tabular}

${ }^{*}$ ) Animais capturados na mata e nas entradas das cavernas; ${ }^{* *}$ ) dois animais não foram sexados; ${ }^{* * *}$ ) nove animais não foram sexados. 

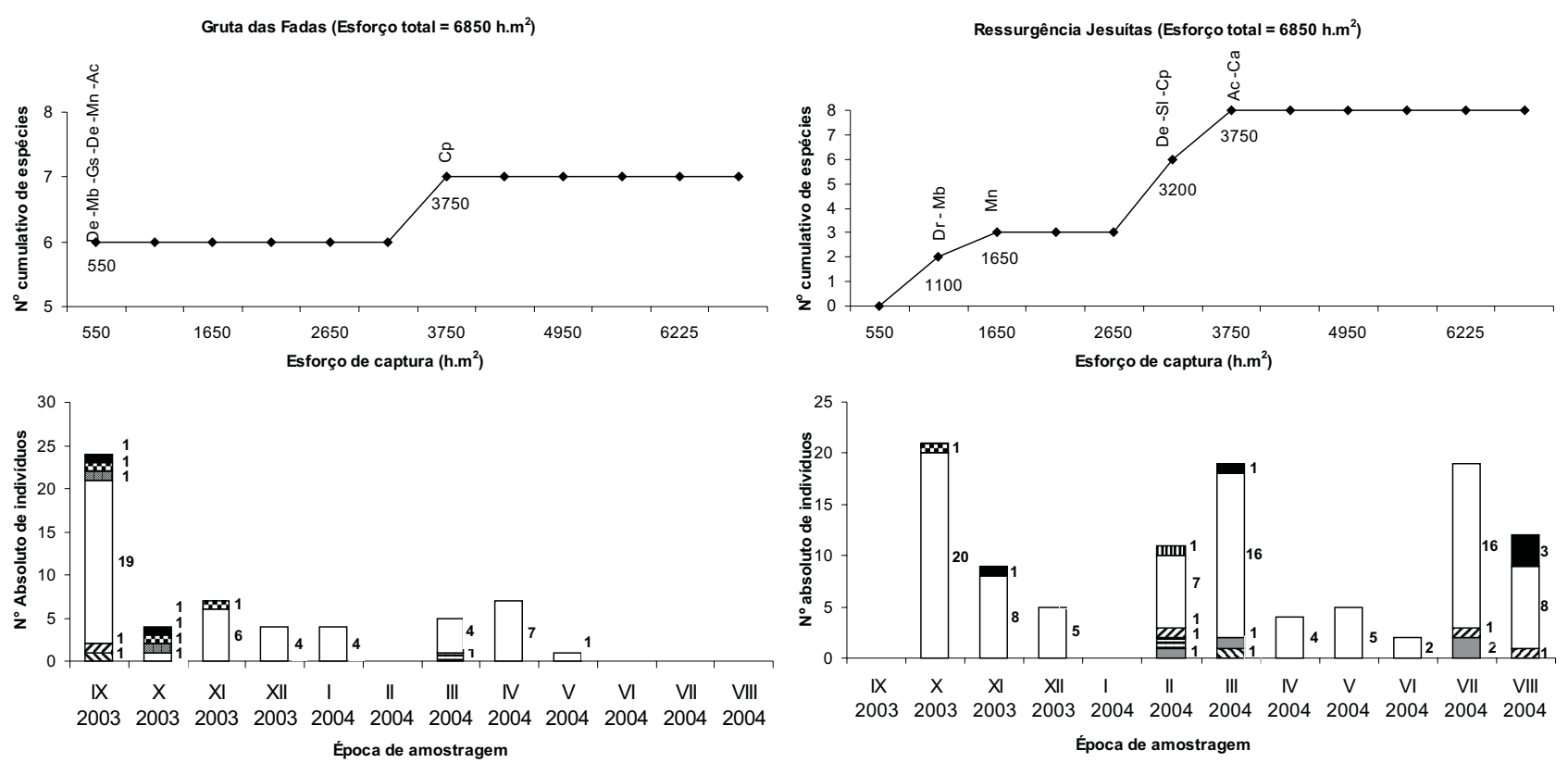

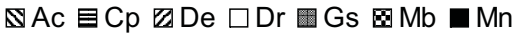
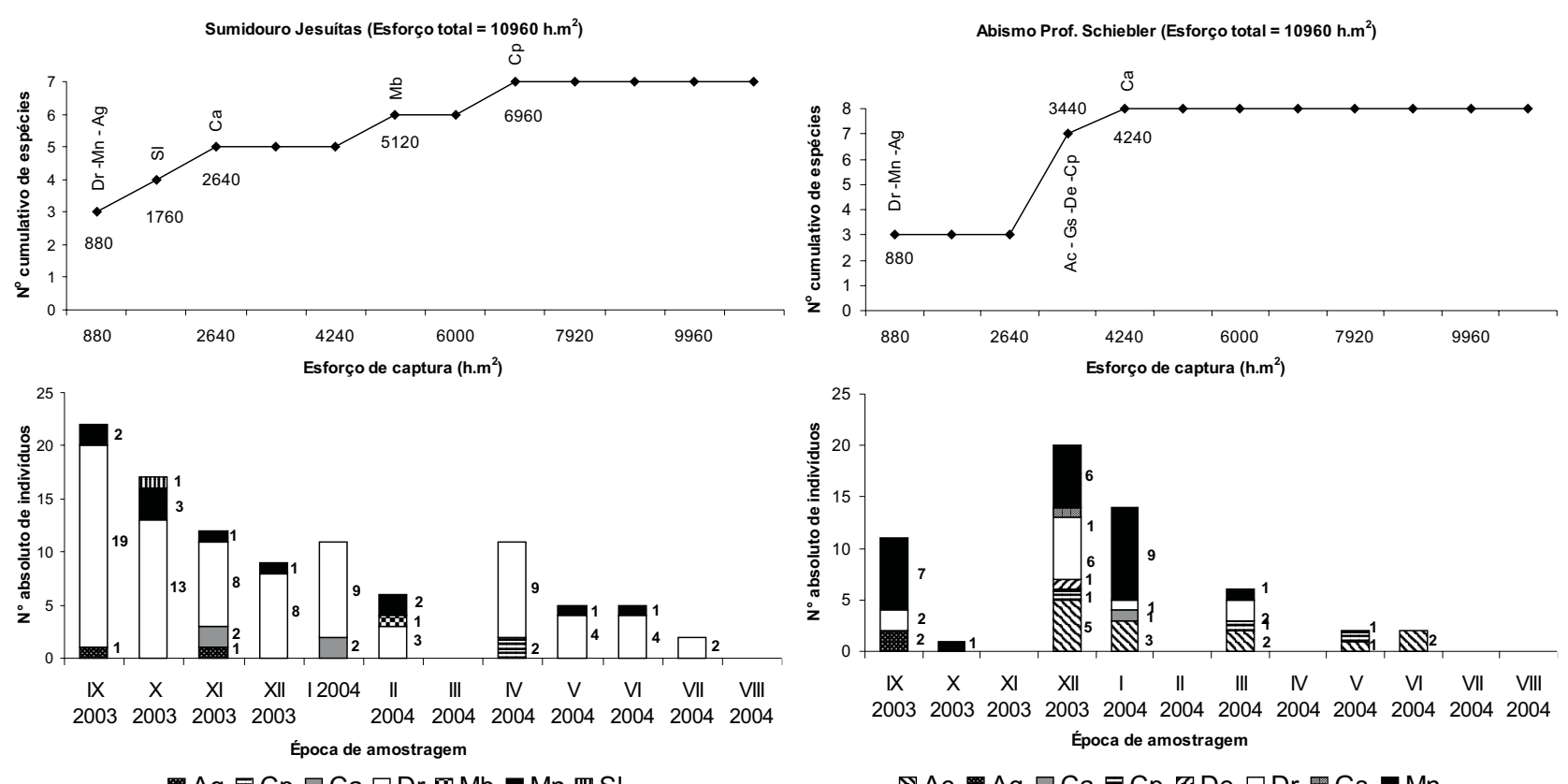

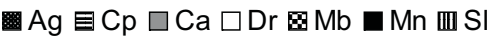

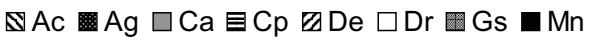

Figura 2. Número de espécies amostradas em cada entrada nos diferentes meses de estudo e número absoluto de espécies acumulado ao longo do ano amostrado. (Ac) Anoura caudifer, (Ag) Anoura geoffroyi, (Ca) Chrotopterus auritus, (Cp) Carollia perspicillata, (De) Diphylla ecaudata, (Dr) Desmodus rotundus, (Gs) Glossophaga soricina, (Mb) Mimon bennettii, (Mn) Myotis nigricans, (SI) Sturnira lilium.

De acordo com Trajano (1984), o número de espécies e o tamanho das populações encontradas em uma caverna podem estar diretamente relacionados com a localização do abrigo, sendo a comunidade mais diversificada quanto maior o núme- ro de espécies na região e quanto mais isolado for este abrigo. Esses morcegos, portanto, ocupariam esses ambientes de forma oportunista, independente das características morfométricas da cavidade. Esta seria uma justificativa plausível para o eleva- 


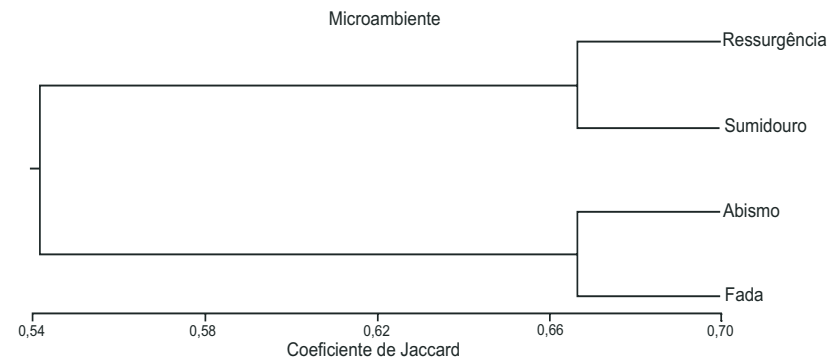

Figura 3. Análise de agrupamento calculada através do Índice de Jaccard, comparando a composição de espécies de morcegos em cada uma das entradas das cavernas no Parque Estadual de Campinhos.
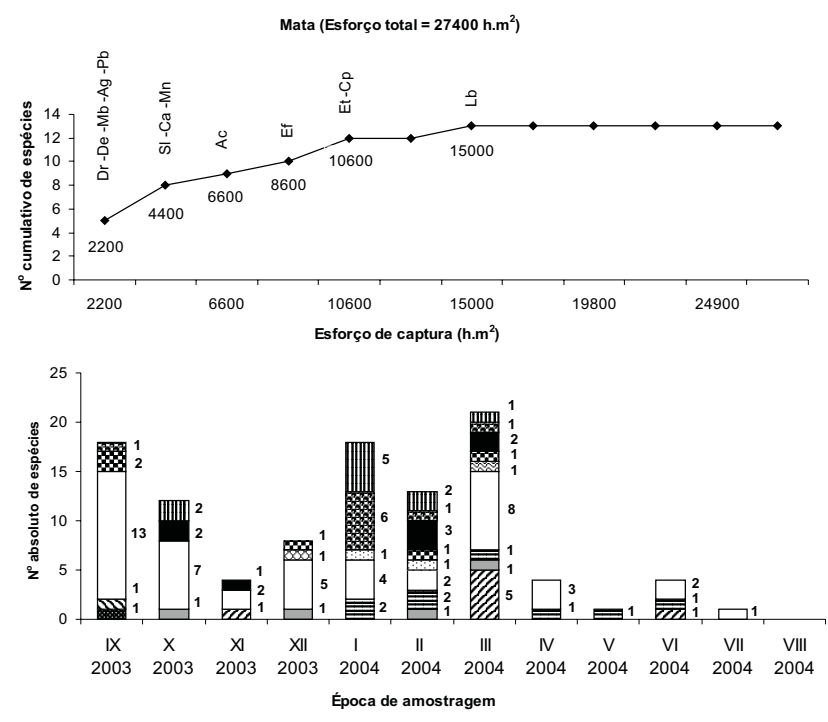

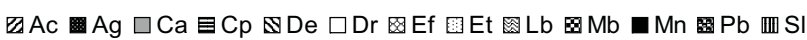

Figura 4. Número de espécies amostradas na mata nos diferentes meses de estudo e número absoluto de espécies acumulado ao longo do ano amostrado. (Ac) Anoura caudifer, (Ag) Anoura geoffroyi, (Ca) Chrotopterus auritus, (Cp) Carollia perspicillata, (De) Diphylla ecaudata, (Dr) Desmodus rotundus, (Ef) Eptesicus furinalis, (Et) Eptesicus taddeii, (Lb) Lasiurus blossevillii, (Mb) Mimon bennettii, (Mn) Myotis nigricans, (Pb) Pygoderma bilabiatum, (SI) Sturnira lilium.

do número de espécies encontradas nas cavernas do PEC. Isso corrobora com os trabalhos de Gregorin \& Mendes (1999), que encontraram 11 espécies no poço Encantado, Bahia (1500 m) e de Trajano \& Gimenez (1998), as quais encontraram 13 espécies para a Gruta Olhos d'Água, Minas Gerais (7000 m). Essas grutas, assim como a do Conjunto Jesuítas/Fada, se encontram isoladas de outras cavernas da região, portanto em áreas com um reduzido número de abrigos.

\section{CONSIDERAÇÕES FINAIS}

No presente estudo, a maioria das espécies de morcegos amostradas no Parque Estadual de Campinhos utilizam as cavernas do Conjunto Jesuítas/Fada como abrigo diurno. A ocorrência de quatro espécies ameaçadas no Estado utilizando esses ambientes indica se tratar de um importante sítio para a conservação desses animais. Os dados apresentados refletem a importância da preservação das cavernas e de estudos nesses ambientes, um hábitat capaz de abrigar espécies importantes da quiropterofauna do Estado. O conhecimento da fauna de quirópteros que utilizam cavernas atinge apenas $11 \%$ do total de cavernas no Estado.

A atividade de controle de hematófagos, no caso de $D$. rotundus e D. ecaudata, como prevenção à raiva é feita tradicionalmente na região sem o acompanhamento de estudos, o que pode levar a um desequilíbrio das populações de animais que dependem do guano dos morcegos como aporte de energia. A realização dessa atividade conflita com os propósitos da Unidade de Conservação, uma vez que sua finalidade é proteger os espécimes dentro da área, podendo ser feito um manejo da fauna após estudos. Deve-se ressaltar a importância dos morcegos como importadores de alimentos para o ambiente cavernícola dado que a importação dos nutrientes nesse ambiente ocorre de maneira inconstante, como carreamento de detritos vegetais e animais por cursos d'água, através de fendas, e outras comunicações com o meio externo (BARR 1967).

Os resultados obtidos têm implicações positivas para a conservação da área, uma vez que o estudo da fauna de quirópteros do Parque poderá contribuir para futuras ações como programas para o manejo no PEC, e ainda auxiliar as ações da Secretaria da Agricultura e do Abastecimento do Estado nas questões relativas ao controle dos hematófagos, o qual poderia afetar todas as outras espécies de morcegos cavernícolas dessa comunidade.

\section{AGRADECIMENTOS}

Ao IAP e IBAMA pela autorização e licença de coleta na área. Ao CNPq pela Bolsa PIBIC cedida ao primeiro autor e pela Bolsa de Produtividade ao segundo autor (Processo 306194/20066). A Atenisi P. Leite e Therys M. Sato pela confirmação e identificação dos exemplares depositados na coleção. A dois revisores anônimos e a João M.D. Miranda pela leitura crítica e sugestões ao manuscrito. Ao GEEP-Açungui pelo apoio logístico e dos espeleólogos Luís F.S. da Rocha, Flavia F. de Lima, Gisele Sessegolo, Emílio T. Osato, Pollyana A. Born e Carol R.C. Muller. À Secretaria de Agricultura e do Abastecimento, Elizete de Oliveira e Marlene Dunaiski. A Gustavo Graciolli, Samira Lonardoni, Simone D. Rosa, Carolina Scultori, Rodrigo Barbosa, Vitor Zwiener, Fernanda Cardoso e Daniel Carneiro.

\section{REFERÊNCIAS BIBLIOGRÁFICAS}

Barquez, R.M.; N.P Giannini. \& M.A. Mares. 1993. Guide to the Bats of Argentina/Guia de los Murcielagos de Argentina. Norman, Oklahoma Museum of Natural History, VIII+119p. 
BARr, T.C. 1967. Observations on ecology of caves. The American Naturalist 101 (922): 475-491.

Bredt, A.; W. Uieda \& E.D. Magalhães. 1999. Morcegos cavernícolas da região do Distrito Federal, centro-oeste do Brasil (Mammalia, Chiroptera). Revista Brasileira de Zoologia 16 (3): 731-770.

CAmpanhã, R.A.C. \& H.G. Fowler. 1995. Movents patterns and roots of the vampire bat Desmodus rotundus in the interior of São Paulo State. Naturallia 20: 191-194

FAbí́n, M.E.; A.M. Rui \& K.P. Oliveira. 1999. Distribuição geográfica de morcegos phyllostomidae (Mammalia: Chiroptera) no Rio Grande do Sul. Iheringia, Série Zoologia (87): 143156.

FonseCA, G.A.B.; G. HeRrManN \& Y.L.R. Leite. 1999. Macrogeography of Brazilian mammals, p. 549-563.In: J.F. EISENBERG \& K.H. REDFORD (Eds). Mammals of the Neotropics: the central neotropics. Chicago, the University of Chicago Press, vol. 3, 609 p.

Geep-Açungui \& Iap. 2003. Plano de Manejo do Parque Estadual de Campinhos. Curitiba, GEEP-Açungui, 314p.

Gomes, M.N. \& W. Uieda. 2004. Abrigos diurnos, composição de colônias, dimorfismo sexual e reprodução do morcego hematófago Desmodus rotundus (E. Geoffroy) (Chiroptera, Phyllostomidae) no Estado de São Paulo, Brasil. Revista Brasileira de Zoologia 21 (3): 629-638.

GREgorin, R. \& L.F. MENDEs. 1999. Sobre quirópteros (Emballonuridae, Phillostomidae, Natalidae) de duas cavernas da Chapada da Diamantina, Bahia, Brasil. Iheringia, Série Zoologia (86): 121-124.

Humphrey, S.R. \& F.J. Bonaccorso. 1979. Population and community ecology, p. 409-441. In R.J. BAKER; J.K. JONEs JR. \& D.C. CARTER (Eds). Biology of the bats of the New World family Phyllostomatidae, part III. Special Publications Museum, Texas Tech University 16: 1-441.

IAPAR 2006. Instituto Agronômico do Paraná. Cartas Climáticas do Paraná. Disponível na World Wide Web em: http:// www.iapar.br/Sma/Cartas_Climaticas/Classificacao_Climatica.htm [acessado em 02/VIII/2006].

IAP. 2000. Plano de Manejo do Parque Estadual de Campinhos. Curitiba, Instituto Ambiental do Paraná, 17p.

Kunz, T.H. 1982. Roosting Ecology of bats, p. 1-55. In: T.H. KunZ (Ed). Ecology of bats. New York, Plenum Press, XVIII+425p.

LAVAL, R.K. 1973. A revision of the neotropical bats of the genus Myotis. Science Bulletin of the Natural History Museum Los Angeles County 15: 1-53.

Lim, B.K. \& M.D. Engstrom. 2001. Species diversity of bats (Mammalia: Chiroptera) in Iwokrama Forest, Guyana, and the Guianan subregion: implications for conservation. Biodiversity and Conservation 10: 613-657.

Margarido, T.C.C. \& F.G. Braga. 2004. Mamíferos, p. 27-142. In: S.B. Мiкich \& R.S. BERNiLs (Eds). Livro vermelho da fauna ameaçada no Estado do Paraná. Curitiba, Instituto Ambiental do Paraná, XVI+763p.
Miranda, J.M.D.; R.F. Moro Rios; A.P. Leite \& F.C. Passos. 2006a. Primeiro registro de Histiotus montanus para o estado do Paraná, sul do Brasil. Revista Brasileira de Zoologia 23 (2): 584-587.

Miranda, J.M.D.; I.P. Bernardi \& F.C. Passos. 2006b. A new species of Eptesicus (Mammalia: Chiroptera: Vespertilionidae) from the Atlantic Forest, Brazil. Zootaxa 1383: 57-68.

Miranda, J.M.D.; A.P. Leite; I.P. Bernardi \& F.C. Passos. 2007. Primeiro registro de Myotis albescens (É. Geoffroy, 1806) (Chiroptera, Vespertilionidae) para o Estado do Paraná, Brasil. Biota Neotropica 7 (1): 13-15.

Miretzki, M. 2003. Morcegos do Estado do Paraná, Brasil (Mammalia, Chiroptera): riqueza de espécies, distribuição e síntese do conhecimento atual. Papéis Avulsos Zoologia 43 (6):101-138.

Oliveira, K.L. \& E.A.B. SipInski. 2001. Mamíferos de quatro sistemas cársticos da Região Metropolitana de Curitiba, PR. Conservando cavernas: 15 anos de espeleologia GEEPAçungui. Curitiba, GEEP-Açungui, 214p.

Passos, F.C. \& G. Graciolli. 2004. Diet observations of Artibeus lituratus (Olfers) (Chiroptera, Phyllostomidae) in two areas of southern Brazil. Revista Brasileira Zoologia 21 (3): 487 489.

Passos, F.C.; W.R. Silva; W.A. Pedro \& M.R. Bonin. 2003. Frugivoria em morcegos (Mammalia, Chiroptera) no Parque Estadual Intervales, sudeste do Brasil. Revista Brasileira de Zoologia 20 (3): 511-517.

Peracchi, A.L; I.P. Lima; N.R. Reis; M.R. Nogueira \& H.O. Filho. 2006. Ordem Chiroptera, p. 155-220. In: N.R. ReIs; A.L. PeraCchi; W.A. Pedro \& I.P. Lima (Eds). Mamíferos do Brasil. Londrina, Nélio R. Reis, 437p.

PinTO-DA-RochA, R. 1995. Sinopse da fauna cavernícola do Brasil. Papéis Avulsos Zoologia 39 (6): 153-173.

Reis, N.R. dos; M.F. Müeller; E.S. Soares; \& A.L. Peracchi. 1993. Lista e chave de quirópteros do Parque Estadual Mata do Godoy e arredores, Londrina, PR. Semina, Ciências Biológicas/Saúde 14 (2): 120-126.

Reis, N.R.; A.L. Peracchi; W.A. Pedro \& I.P. Lima. 2006. Mamíferos do Brasil. Londrina, 437p.

Sessegolo, G.C.; D.P. Zakrzewski; V. Theulen \& L.F. Silva-da RoCHA. 1996. A degradação ambiental de cavidades naturais subterrâneas da Região Metropolitana de Curitiba-PR, p. 58. In: G.C. Sessegolo; L.F. Silva-da Rocha \& V. Theulen (Eds). Cavernas do Paraná: dez anos de espeleologia - GEEPAçungui. Curitiba, GEEP-Açungui, 34p.

Silva, S.S.P.; P.G. Guedes \& A.L. Peracchi. 2001. Levantamento preliminar dos morcegos do Parque Nacional de Ubajara (Mammalia, Chiroptera), Ceará, Brasil. Revista Brasileira de Zoologia 18 (1): 139-144.

Simmons N.B. 2005. Order Chiroptera, p. 312-529. In: D.E. WiLSON \& D.M. REEDER (Eds). Mammal species of the world: a taxonomic and geographic reference. Baltimore, Johns Hopkins University Press, vol.1, $3^{\text {rd }}$ ed., 2142p. 
Straube, F.C. \& G.V. Bianconi. 2002. Sobre a grandeza e a unidade utilizada para estimar esforço de captura com utilização de redes-de-neblina. Chiroptera Neotropical 8 (1-2): 150152.

Trajano, E. 1984. Ecologia de populações de morcegos cavernícolas em uma região cárstica do sudeste do Brasil. Revista Brasileira de Zoologia 2 (5): 255-320.

Trajano, E. 1995. Protecting caves for bats or bats for the caves? Chiroptera Neotropical 1 (2): 19-22.

Trajano, E. 1996. Movements of Cave Bats in Southeastern Brazil, with Emphasis on population Ecology of Commom Vampire Bat, Desmodus rotundus (Chiroptera). Biotropica 28 (1): 121-129.

Trajano, E. \& E. Gimenez. 1998. Bat community in a cave from Eastern Brazil, Including a New record of lionycteris (Phyllos- tomidae, Glossiphaginae) Studies on Neotropical Fauna and Environment 33: 69-75.

Veloso, H.P.; A.L.R. Rangel Filho \& J.C.A. Lima. 1991. Classificação da vegetação brasileira adaptada a um sistema universal. Rio de Janeiro, IBGE, 123p.

VizotTo, L.D. \& V.A. TADDEI. 1973. Chave para determinação de quirópteros brasileiros. São José do Rio Preto, Gráfica Francal, 72p.

Zeppelini, D.F.; A.C. Ribeiro; G.C. Ribeiro; M.P.A. Fracasso; M.M. PaVanni; O.M.P. Oliveira; S.A. Oliveira \& A.C. Marques. 2003. Faunistic survey of sandstone caves from Altinópolis region, São Paulo State, Brazil. Papéis Avulsos Zoologia 43 (5): 93-99.

Willig, M.R. \& K.W. Selcer. 1989. Bat species density gradients in the New World - a statistical assessment. Journal of Biogeography 12: 189-195.

Recebido em 23.VIII.2006; aceito em 13.VII.2007. 